\title{
An Integrated Approach to Detection of Mycobacterium Tuberculosis Using BD MGIT 320 System and other Diagnostic Modalities
}

\author{
Gargi Choudhury*, Partha Pratim Das, Lahari Saikia \\ Department of Microbiology, Assam Medical College, Dibrugarh, India
}

\begin{abstract}
Background: Application of automated liquid culture system like Becton, Dickinson and Company (BD) MGIT 320 can shorten the recovery time of Mycobacteria in clinical diagnostic laboratories as compared to Lowenstein Jensen (LJ) medium. The present study aimed to compare the results of BD MGIT 320 culture system with that of LJ medium and also to evaluate the usefulness of molecular methods as an adjunct.

Methods: Specimen from clinically suspected cases of tuberculosis were processed as per standard guidelines for smear microscopy, solid culture in LJ media, liquid culture in BD MGIT 320 system and Line probe assay. Polymerase chain reaction was performed on extracted DNA from MGIT positive specimen to detect IS6110 and MPB64 gene targets.

Result: Out of total 83 specimens, a statistically significant higher isolation rate $(25.3 \%, \mathrm{n}=21)$ was shown by MGIT 320 system in comparison to LJ medium $(12.04 \%, n=10)$. Line Probe Assay was positive in $37.3 \%(n=31)$ and direct microscopy was positive in only $13.25 \%$ ( $n=11)$ specimen. The average duration of detection was found to be more rapid by MGIT320 than LJ culture (18 days vs 24.1 days). Among the 21 MGIT positive specimens 7 were found positive for both IS6110 and MPB64 gene while 7 specimens were found positive only for IS6110.
\end{abstract}

Conclusion: The automated liquid culture systems like BD MGIT 320 have a higher isolation rate and are more rapid as compared to solid media. But adoption of Line probe assay combined with MGIT culture will definitely yield rapid and efficient detection of Mycobacteria.

Keywords: Liquid Culture, Multi Drug Resistant Tuberculosis, Extrapulmonary Tuberculosis, Line Probe Assay.

\section{Introduction}

The increasing incidence of tuberculosis (TB) and emergence of multidrug resistant tuberculosis (MDRTB) has made it essential for health care laboratories to detect Mycobacteria rapidly along with drug susceptibility pattern. In 2015, the world had an estimated 10.4 million new TB cases; India recorded an incidence of 2.84 million with 0.13 million drug resistant TB cases.[1] As conventional culture methods are cumbersome and need longer incubation, adoption of automated culture system like BD (Becton, Dickinson and Company) MGIT (Mycobacterium Growth Indicator Tube) 320 liquid culture system can shorten the recovery time of Mycobacteria.[2] The MGIT TBc identification test based on detection of MPT64, a protein secreted by Mycobacterium tuberculosis (MTb) complex can detect MTb complex in 15 minutes from positive MGIT cultures. Liquid culture shortens the time of detection in comparison to solid culture but it is still not a rapid test as labor intensive biochemical methods are required for species identification and it takes longer incubation for smear-negative specimens.[3,4,5] In recent times, laboratories are adopting nucleic acid amplification (NAA) diagnostic technologies owing to their rapidity and sensitivity. Methods like Line probe assay (LPA) or Polymerase Chain Reaction (PCR) assay as adjunct to detect MTb complex from clinical specimen can overcome the limitations of liquid culture.

The present study aimed to compare the results of $\mathrm{BD}$ MGIT 320 culture system with that of Lowenstein-Jensen (LJ) medium for recovery rates and the mean time required to detect Mycobacteria from clinical specimens. Our study also aimed to evaluate the usefulness of molecular methods as an adjunct for rapid detection.

\section{Materials and Methods}

The study involves specimens from clinically suspected cases of tuberculosis (both pulmonary and extrapulmonary) received in Mycobacteriology laboratory for detection of Mycobacteria during a period of 18 months from September, 2015 to February, 2017.

Specimen Collection and Processing: A total number of 83 clinical specimens were collected after obtaining prior consent from the patients and processed in bio-safety level (BSL)-3 laboratories in the Department of Microbiology. Specimens were digested and decontaminated with an equal volume of $\mathrm{N}$-acetyl- L-cysteine (NALC)-NaOH (final 
concentration $2 \% \mathrm{NaOH}$ and $0.5 \%$ NALC) for 15 minutes at room temperature and then neutralized with sterile 0.067 $\mathrm{M}$ phosphate buffer ( $\mathrm{pH}$ 6.8).[6] After centrifugation at $3000 \mathrm{~g}$ for 15 minutes, the sediment was re-suspended in 2.0 $\mathrm{mL}$ of phosphate buffer. An aliquot of the suspension was used for Ziehl Neelsen (ZN) stain to detect acid-fast bacilli (AFB); $0.5 \mathrm{~mL}$ of the suspension was inoculated into a BD MGIT 320 tube, and another $0.2 \mathrm{~mL}$ was inoculated onto one Lowenstein-Jensen (LJ) slant (Himedia lab, Mumbai). Another aliquot of the suspension was used for molecular identification by HAINS MTBDRplus Line Probe Assay as well as conventional PCR assay for IS6110 and MPB64 gene targets.

LJ Culture: After inoculation into liquid media, $0.2 \mathrm{ml}$ of each processed specimen was also inoculated onto the surface of an LJ slant procured from Himedia Laboratory Pvt. Ltd, Mumbai and the cultures were incubated at 37 degree for 8 weeks. The LJ medium was examined on alternate days from day 2 to day 7 and once a week from day 8 to day 56 according to established laboratory procedures.[7]

BD MGIT 320 Culture: [8] Principle: MGIT tube contains Middlebrook 7H9 liquid media and an oxygen quenched fluorochrome embedded in silicon at the bottom of the tube. During bacterial growth, the free oxygen is utilized and replaced with carbon-dioxide. With the depletion of free oxygen the fluorochrome is no longer inhibited resulting in fluorescence within the MGIT tube when visualized under UV light. Instrument declares a tube negative if it remains negative for six weeks (42 days).

Inoculation of MGIT medium: After labeling MGIT tube with specimen number, $0.8 \mathrm{~mL}$ of Growth Supplement/ MGIT PANTA (polymyxin, amphotericin B, nalidixic acid, trimethoprim, and azlocillin) antibiotic mixture was added just prior to inoculation of $0.5 \mathrm{~mL}$ of the suspension into a BD MGIT 320 tube. After proper mixing, tubes were entered into the instrument and allowed for automatic testing for the duration of the recommended 42 days. Positive MGIT tubes were processed for the MGIT TBc identification test; positive result of which confirmed growth of Mtb complex.

Line Probe Assay (LPA): The GenoType MTBDRplus VER 2.0 (Hain Lifesciences GmBH, Nehren, Germany) is a commercially available line probe assay based on multiplex PCR combined with reverse hybridization on nitrocellulose strips, targeting common mutations and was performed according to the manufacturer's instructions [9]. This assay identifies Rifampicin (RIF) and Isoniazid (INH) resistance by detecting the most common mutations of the rpoB gene and the $k a t G$ and $i n h A$ genes, respectively, and can be used on direct specimens as well as culture isolates. Mycobacterial DNA was extracted in BSL-3 laboratory according to manufacturer's instructions.[9]

Conventional PCR : Multiplex PCR assay was performed for identification of $M$. tuberculosis using specific pair of primers designed to amplify IS6110 and MPB64 gene in the Mtb complex and the expected band size was $123 \mathrm{bp}$ for IS6110 and $240 \mathrm{bp}$ for MPB64. [10] The sequences of primer used were IS6110F: 5'-CCTGCGAGCGTAGGCGT-3', IS6110R: 5'-CTCGTCCAGCGCCGCTTCGG-3', MPB64F: 5'-TCCGCTGCCAGTCGTCTTCC-3', MPB64R: 5'- GTCCTCGCGAGTCTAGGCCA-3' .[10] Briefly, following components were added to a sterile eppendorf tube (for $20 \mu \mathrm{L}$ reaction volume)- $10 \mu \mathrm{L}$ universal PCR mix 2X (Promega), $2 \mu \mathrm{L}$ of each of the forward and reverse primers $(10 \mathrm{pm} / \mu \mathrm{L}), 1 \mu \mathrm{L}$ of template and rest nuclease free water. The positive control included was the DNA of $M$. tuberculosis H37Rv strain and negative control was the PCR grade water. DNA amplification was performed for 40 cycles following an initial denaturation step at $95^{\circ} \mathrm{C}$ for 5 minutes, denaturation at $94^{\circ} \mathrm{C}$ for 1 minute, annealing at $65^{\circ} \mathrm{C}$ for 1.5 minutes, extension at $72^{\circ} \mathrm{C}$ for 1.5 minutes and final extension at $72^{\circ} \mathrm{C}$ for 10 minutes followed by storage at $4^{\circ}$ Celsius until detection. The amplified products were then run on $1.5 \%$ agarose gel electrophoresis stained with ethidium bromide using $100 \mathrm{bp}$ ladder followed by detection of target band using gel documentation system (BIORAD) (Fig 1).

Quality control of all the methods was performed using $M$. tuberculosis, H37Rv strain.

\section{Result}

Out of 83 clinical specimens (pulmonary $=38$, extrapulmonary $=45)$ processed, a total of $21(25.3 \%)$ were found positive for MTb complex by the BD MGIT 320 culture systems, confirmed by MGIT TBc identification test (pulmonary $=8$, extra-pulmonary $=13$ ) (Table 1 ). Conventional culture in LJ medium showed growth of Mycobacteria in $10(12.04 \%)$ number of specimen (pulmonary $=3$, extra-pulmonary $=7$ ). Initially HAINS Genotype MTBDRplus (LPA) was positive in 31 (37.3\%) number of specimen while direct microscopy (ZN Stain) for acid fast bacilli was positive only in 11 (13.25\%) numbers. Among the MGIT positive specimens, sputum and pus shared the top of the list ( 5 nos. each) followed by 4 nos. of tissue specimen (Table 1).

All MGIT positive specimens (except one pleural fluid) were initially found positive for MTb complex by LPA, of which only 8 specimens were positive on direct microscopy. The average duration of MGIT positivity was 
found to be 18 days while LJ media showed an average duration of 24.1 days for positive growth (Table 2). In case of pulmonary specimens, the mean recovery time was 17.3 days whereas in extra-pulmonary specimens it was found to be 18.9 days by the BD MGIT 320 TB system in the present study.

Multiplex PCR of all MGIT positive specimens showed positive result for both $I S 6110$ and MPB64 gene in 7 numbers, negative for both the targets in another 7, while 7 specimens were found positive only for IS6110 (Table 2).

Drug Susceptibility: Results of LPA positive specimens showed that 12 were susceptible for both Rifampicin (RIF) and Isoniazid (INH), 3 were indeterminate and 3 were resistant to both the drugs while one specimen was susceptible to Rifampicin and indeterminate to Isoniazid; yet another was Isoniazid monoresistant.

Table 1: Distribution of clinical specimen and result of different assay.

\begin{tabular}{|l|c|c|c|c|c|} 
Sample type & $\begin{array}{c}\text { No. of sample } \\
\text { processed } \\
(\mathbf{n = 8 3})\end{array}$ & $\begin{array}{c}\text { ZN stain result } \\
(\mathbf{n = 1 1 )}\end{array}$ & $\begin{array}{c}\text { MTBDR plus (LPA) } \\
\text { Positive } \\
(\mathbf{n = 3 1 )}\end{array}$ & $\begin{array}{c}\text { MGIT culture } \\
\text { Positive } \\
(\mathbf{n = 2 1 )}\end{array}$ & $\begin{array}{c}\text { LJ Culture } \\
\text { Positive } \\
(\mathbf{n}=\mathbf{1 0})\end{array}$ \\
\hline Sputum & 18 & 2 & 7 & 5 & 2 \\
\hline Pus & 17 & 5 & 8 & 5 & 3 \\
\hline BAL & 10 & 0 & 4 & 2 & 0 \\
\hline Pleural fluid & 10 & 0 & 2 & 1 & 1 \\
\hline Ascitic fluid & 5 & 0 & 1 & 0 & 0 \\
\hline Urine & 7 & 3 & 3 & 2 & 0 \\
\hline CSF & 5 & 0 & 2 & 2 & 0 \\
\hline Tissue & 10 & 1 & 4 & 4 & 4 \\
\hline Bone marrow & 1 & 0 & 0 & 0 & 0 \\
\hline
\end{tabular}

Table 2: Results of other diagnostic methods in MGIT positive cases.

\begin{tabular}{|c|c|c|c|c|c|c|c|c|c|c|c|}
\hline \multirow{2}{*}{ Patient ID } & \multirow{2}{*}{ Age } & \multirow{2}{*}{ Gender } & \multirow{2}{*}{$\begin{array}{c}\text { Type of } \\
\text { Specimen }\end{array}$} & \multirow{2}{*}{\begin{tabular}{|c|} 
Direct \\
Microscopy \\
(AFB) result
\end{tabular}} & \multirow{2}{*}{$\begin{array}{c}\text { Line Probe } \\
\text { Assay } \\
\text { result }\end{array}$} & \multirow{2}{*}{$\begin{array}{l}\text { MGIT } \\
\text { culture } \\
\text { result }\end{array}$} & \multirow{2}{*}{$\begin{array}{l}\text { Time to } \\
\text { MGIT } \\
\text { Positivity }\end{array}$} & \multirow{2}{*}{$\begin{array}{l}\text { LJ } \\
\text { Culture } \\
\text { Result }\end{array}$} & \multirow{2}{*}{$\begin{array}{c}\text { Time } \\
\text { to LJ } \\
\text { Positivity }\end{array}$} & \multicolumn{2}{|c|}{ PCR result } \\
\hline & & & & & & & & & & IS6110 & MPB64 \\
\hline 106 & 54 & Female & Pus & Neg & Pos & Pos & 12 days & Neg & - & Pos & Pos \\
\hline 107 & 54 & Female & Tissue & $\mathrm{Neg}$ & Pos & Pos & 15 days & Pos & 21 days & $\mathrm{Neg}$ & $\mathrm{Neg}$ \\
\hline 111 & 20 & Male & Sputum & $\mathrm{Neg}$ & Pos & Pos & 13 days & Neg & - & $\mathrm{Neg}$ & Neg \\
\hline 145 & 30 & Female & Pus & Pos & Pos & Pos & 14 days & Neg & - & Pos & Pos \\
\hline 157 & 25 & Female & Sputum & Pos & Pos & Pos & 13 days & $\mathrm{Neg}$ & - & Pos & $\mathrm{Neg}$ \\
\hline 158 & 25 & Female & Sputum & $\mathrm{Neg}$ & Pos & Pos & 21 days & Neg & - & Pos & $\mathrm{Neg}$ \\
\hline 167 & 18 & Female & Pus & Pos & Pos & Pos & 14 days & Pos & 23 days & Pos & $\mathrm{Neg}$ \\
\hline 178 & 48 & Male & $\begin{array}{l}\text { Pleural } \\
\text { Fluid }\end{array}$ & Neg & Neg & Pos & 15 days & Pos & 18 days & Pos & Neg \\
\hline 185 & 28 & Female & Urine & Pos & Pos & Pos & 16 days & $\mathrm{Neg}$ & - & Pos & $\mathrm{Neg}$ \\
\hline 192 & 10 & Female & Pus & Pos & Pos & Pos & 34 days & Pos & 15 days & $\mathrm{Neg}$ & Neg \\
\hline 219 & 35 & Female & Urine & Pos & Pos & Pos & 18 days & $\mathrm{Neg}$ & - & Pos & Pos \\
\hline 224 & 23 & Male & Tissue & Pos & Pos & Pos & 17 days & Pos & 24 days & $\mathrm{Neg}$ & $\mathrm{Neg}$ \\
\hline 226 & 65 & Male & BAL & $\mathrm{Neg}$ & Pos & Pos & 22 days & $\mathrm{Neg}$ & - & $\mathrm{Neg}$ & $\mathrm{Neg}$ \\
\hline 227 & 25 & Male & BAL & $\mathrm{Neg}$ & Pos & Pos & 22 days & $\mathrm{Neg}$ & - & $\mathrm{Neg}$ & $\mathrm{Neg}$ \\
\hline 245 & 28 & Male & Sputum & $\mathrm{Neg}$ & Pos & Pos & 18 days & Pos & 24 days & Pos & Pos \\
\hline 320 & 28 & Male & Sputum & Pos & Pos & Pos & 15 days & Pos & 27 days & Pos & Pos \\
\hline 339 & 11 & Female & Pus & $\mathrm{Neg}$ & Pos & Pos & 30 days & Pos & 18 days & $\mathrm{Neg}$ & $\mathrm{Neg}$ \\
\hline 357 & 24 & Male & CSF & $\mathrm{Neg}$ & Pos & Pos & 20 days & Neg & - & Pos & $\mathrm{Neg}$ \\
\hline 367 & 24 & Male & Tissue & $\mathrm{Neg}$ & Pos & Pos & 27 days & Pos & 31 days & Pos & Pos \\
\hline 482 & 7 & Male & Tissue & $\mathrm{Neg}$ & Pos & Pos & 14 days & Pos & 40 days & Pos & Pos \\
\hline 531 & 11 & Male & CSF & $\mathrm{Neg}$ & Pos & Pos & 15 days & $\mathrm{Neg}$ & - & Pos & $\mathrm{Neg}$ \\
\hline
\end{tabular}

Foot Note: Pos-Positive; Neg-Negative 


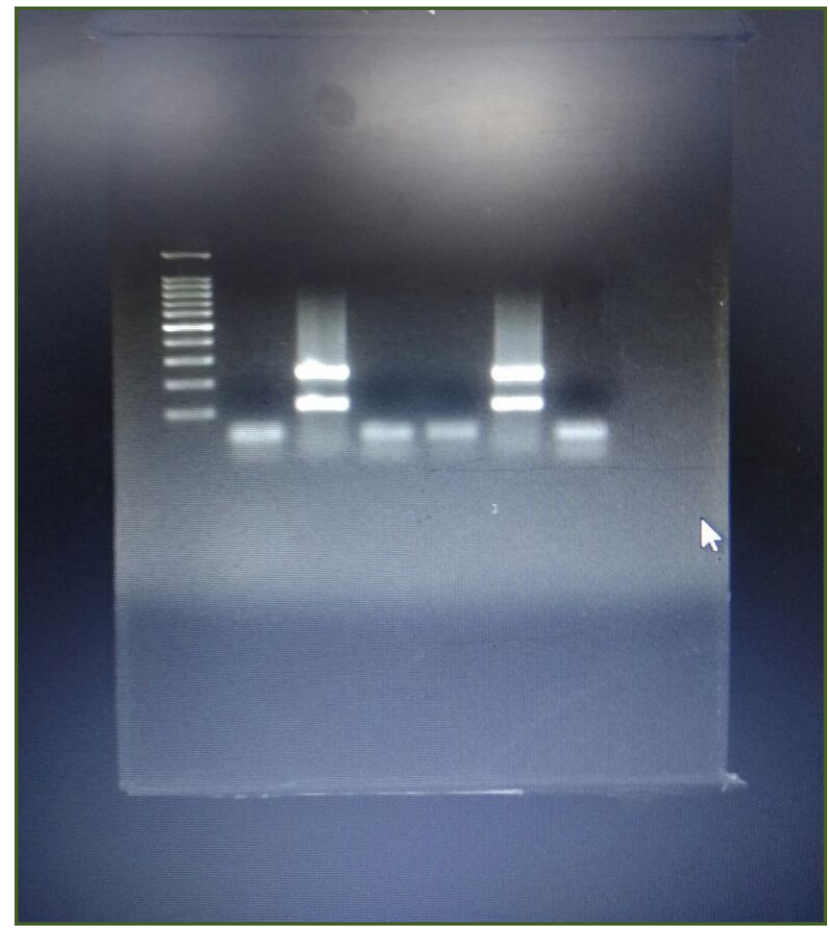

Fig. 1: Multiplex PCR for IS6110 and MPB64 gene; Starting from left: Lane 1- 100 bp ladder; Lane 2,4,5 - PCR negative specimen; Lane 3- specimen positive for IS6110 (123 bp) and MPB64 (240 bp); Lane 6- positive control (H37Rv strain), Lane 7- Negative control.

\section{Discussion}

The increased incidence of tuberculosis worldwide has made rapid and efficient culture strategies very essential which can easily be adopted in a clinical Mycobacteriology laboratory. Solid egg based medium like LJ medium is the current "gold standard" culture method for detection of Mycobacteria [6,11]. One of the culture methods, MGIT liquid culture system is easy to handle, non-radiometric, and at present does not need costly instrumentation. The BD MGIT 320 culture system contains only one drawer, holds 320 MGIT tubes and can be placed on a bench top. It is designed for laboratories with limited space and workload. MGIT 320 can be used by a laboratory that cultures up to 40 Mycobacteriology specimens per week, with an incubation period of 6 weeks, and performs up to five drug susceptibility tests per week. [2]

Our study compared BD MGIT 320 system with LJ culture method for Mycobacteria and evaluated following important parameters - the rate of recovery and mean time to detection. Combined use of liquid and solid media is essential in good laboratory practice for successful isolation of mycobacterium which we followed in our study. The present study showed higher isolation rate in BD MGIT 320 system than LJ culture (25.3\% vs $12.04 \%$ ) and was found statistically significant ( $\mathrm{p}$ value $=<0.05$ using Chi square test). The obtained results are in agreement with those reported by Hanna et al, Tortoli et al which showed that the MGIT system had a recovery rate higher than in solid media.[3,12] Dongsi and Dunne also found that the MGIT culture system consistently gave better isolation rates of all Mycobacterium species from a variety of clinical specimens than the traditional L.J. slants.[13] Rishi S et al reported that isolation rate in MGIT system was higher as compared to solid media (50.6\% vs $33.6 \%$ ) so as Rodrigues et al ( $41 \%$ vs $24 \%$ ).[14,15] In addition, Bunger $\mathrm{R}$ et al also reported that the highest mycobacterial recovery rate was by MGIT (91.6\%) as compared to LJ media (58.3\%).[16]

The present study revealed that the mean time to detection of $M$. tuberculosis complex were 18 days by BD MGIT system and 24.1 days by solid media which is in agreement with an earlier study by Hanna et al (14.4 days by MGIT vs. 24.1 days for solid media).[3] The average time to detection of mycobacterial growth according to the smear positivity was noted to be shorter by MGIT culture in our study (15.3 days for AFB positive and 18.7 days for AFB negative specimen) compared to solid media (22.2 days for AFB positive and 25.3 days for AFB negative specimen). This is further supported by Rishi et al, Bunger et al and Chihota et al.[14,16,17] One smear positive pus specimen took 34 days to detection by MGIT system while another smear negative pus specimen flagged positive on day 30 . Both the isolates were found to be MDRTB by LPA.

Thus, in this study MGIT 320 was found to be more rapid and efficient method than the conventional LJ media. Though culture contamination is often encountered in most of the laboratories, meticulous adherence to the manufacturer's manual and protocols, and rapid transport and/or refrigeration of samples could overcome any such problem in our study.

NAA based diagnostic techniques have the potential to increase the sensitivity as well as to considerably reduce the detection time.[18] Most studies have used IS6110 as a target for PCR-based diagnosis of tuberculosis with variable degrees of success.[19] However, this insertion element is absent in a proportion of Mtb isolates which limits its use as a sole target for the detection of tuberculosis in India. $[20,21]$ Use of two or more gene targets for amplification has been demonstrated to increase the diagnostic yield of MTB infection in some earlier studies.[22,23] We evaluated the role of PCR using two different targets IS6110 and MPB64 specific for MTb complex in MGIT positive cases and found presence of IS6110 in $66.6 \%$ isolates while both IS6110 and MPB64 in 33.3\% and none of the targets amplified in the rest. 


\section{Limitation of The Study}

Though we detected the susceptibility to Rifampicin and Isoniazid using LPA in all cases, we performed MGIT DST only in those isolates which were found resistant/ indeterminate by LPA. Comparison of DST result by both the methods would have been more useful which we intend to follow in future studies. Also due to limited number of cases in the present study it is too early to comment on the usefulness of PCR assay using multiple targets.

\section{Conclusion}

Hence, we conclude that the automated culture systems like BD MGIT 320 have a higher isolation rate as compared to solid media (LJ). But adoption of Line probe assay combined with MGIT culture will definitely yield rapid and efficient detection of Mycobacterium as well as multidrug resistant TB cases.

\section{Acknowledgements}

We thank Mr Lakhi Gogoi, Mr. Dipankar Borah, Department of Microbiology, Assam Medical College, Dibrugarh and Mr. Manash P Borah, Multidisciplinary Research Unit, Assam Medical College, Dibrugarh for their technical assistance. We are grateful to the Department of Biotechnology, Govt. of India for providing us the research platform.

\section{Reference}

1. SEARO WHO, New Delhi, India; 2017 [cited 2017 Sep 25]. Available from: https://www.searo.who.int/topics/ tuberculosis

2. Duque A, Lin SY, Desmond E, Rienthong S, Rienthong D, Boonin C.Evaluation of the BD Bactec MGIT 320 for detection of Mycobacteria and drug susceptibility testing of Mycobacterium tuberculosis. J. Clin. Microbiol 2013;51:34033405. http://dx.doi.org/10.1128/JCM.01357-13

3. Hanna B A, Ebrahimzadeh A, Elliott L B, Morgan M A, Novac S M, Gerdes S R et al. Multicenter evaluation of the BACTEC MGIT 960 system for recovery of mycobacteria. J Clin Microbiol 1999; 37: 748-752. https://www.ncbi.nlm. nih.gov/pmc/articles/PMC84542

4. Somoskovi A, Kodmon C, Lantos A, Bartfai Z, Tamasi L, Fuzy J et al. Comparison of recoveries of Mycobacterium tuberculosisusing the automated BACTEC MGIT 960 system, the BACTEC 460 TB system, and LowensteinJensen medium [Internet]. J Clin Microbiol. 2000 Jun;38(6):2395-2397 [cited 2017 Sep 26] https://www.ncbi. nlm.nih.gov/pmc/articles/PMC86818

5. Dowdy D, Lourenço M, Cavalcante S, Saraceni V, King B, Golub J et al. Impact and Cost-Effectiveness of Culture for Diagnosis of Tuberculosis in HIV-Infected Brazilian Adults. PLoS ONE [Internet]. 2008; 3(12):e4057 [cited 26 September 2017] https://doi.org/10.1371/journal.pone.0004057
6. Kent PT, Kubica GP. Public health mycobacteriology: a guide for the level III laboratory. Atlanta, GA: U.S. Dept. of Health and Human Services, Public Health Service, Centers for Disease Control; 1988.

7. Revised National TB Control Programme. Manual of standard operating procedures for culture of Mycobacterium tuberculosis and drug susceptibility testing on solid medium [Internet], Version No. 01.01, 2009. [cited 16 September 2017] http://tbcindia.nic.in/ WriteReadData/1892s/6995271860Training manual M tuberculosis C DST.pdf

8. Siddiqi, S. H., \& Rüsch-Gerdes, S. For BACTEC ${ }^{\text {TM }}$ MGIT $960^{\mathrm{TM}}$ TB System [Internet] 2006. [cited 16 September 2017] https://www.finddx.org/wp-content/uploads/2016/02/ mgit_manual_nov2006.pdf

9. Hain Lifescience, GmbH, Nehren. Germany. Genotype MTBDRplus TM, version 2.0. Instruction manual [Internet] [cited 2017Sep26] http://www.hainlifescience.com/pdf

10. Sharma K, Sinha S, Sharma A, Prasad K, Rana S, Sharma M, et al. Multiplex PCR for rapid diagnosis of gastrointestinal tuberculosis. [Internet] J Glob Infect Dis 2013;5(2):49-53 [cited 2017 Sep 27] https://www.ncbi.nlm.nih.gov/pmc/ articles/PMC3703210

11. Nolte, F. S., B. Metchock. Mycobacterium. In P. R. Murray, E. J. Baron, M. A. Pfaller, F. C. Tenover, and R. H. Yolken, ed. by. Manual of clinical microbiology. 6th ed. ASM Press, Washington, D.C.; 1995. p. 400-437.

12. Tortoli E, Cichero P, Piersimoni C, Simonetti T, Gesu G, Nistta D. Use of BACTEC MGIT for recovery of mycobacteria from clinical specimens: multicentric study. J Clin Microbiol. 1999;37: 3578- 3582. http://jcm.asm.org/ content/37/11/3578.full.pdf

13. Dongsi L, Bobby H, Dunne W M. Comparison of the automated mycobacteria growth indicator tube system (BACTEC MGIT 960) with Lowenstein Jensen medium for recovery of mycobacteria from clinical specimens. Microbiol Infect Dis. 2002; 118:542-545. https://academic.oup.com/ ajcp/article-pdf/118/4/542/4984895/ajcpath118-0542.pdf

14. Rishi S, Sinha P, Malhotra B, Pal N. A Comparative study for the detection of mycobacteria by BACTEC MGIT 960, Lowenstein Jensen media and direct AFB smear examination. Ind J Med Microbiol. 2007 Oct; 25(4):383386. https://www.ncbi.nlm.nih.gov/pubmed/18087090

15. Rodrigues C, Shenai S, Sadani M, Sukhadia N, Jani M, Ajbani $\mathrm{K}$ et al. Evaluation of the bactec MGIT 960 TB system for recovery and identification of Mycobacterium tuberculosis complex in a high volume tertiary care centre. Indian J Med Microbiol [serial online] 2009; 27:217-221. [cited 2017 Aug 28] http://www.ijmm.org/text.asp?2009/27/3/217/53203

16. Bunger R, Singh VA, Avneet, Mehta S, Pathania D. Evaluation of BACTEC Micro MGIT with Lowenstein Jensen media for detection of Mycobacteria in clinically suspected patients of extra pulmonary tuberculosis in a tertiary care hospital at Mullana (Ambala). J Med Microb 
Diagn. 2013,2:2. https://www.omicsonline.org/evaluationof-bactec-micro-mgit-with-lowenstein-jensen-media-fordetection-of-mycobacteria-in-clinically-suspected-patientsof-extra-pulmonary-tuberculosis-2161-0703.1000123. php?aid=12037

17. Chihota VN, Grant AD, Fielding K, Ndibongo B, Zyl AV, Muirhead D et al. Liquid versus solid culture for tuberculosis: performance and cost in a resource-constrained setting. Int $\mathrm{J}$ Tuberc Lung Dis. 2010;14(8):1024-1031. https://www.ncbi. nlm.nih.gov/pubmed/20626948

18. Horner PJ, Moss FM: Tuberculosis in HIV infection. Int J STD AIDS 1991, 2(3):162-167. https://www.ncbi.nlm.nih. gov/pubmed/1863645.

19. Jatana S. K., Nair M. N., Lahiri K. K., Sarin N. P. Polymerase chain reaction in the diagnosis of tuberculosis. Indian Pediatr. 2000; 37, 375-382. http://indianpediatrics. net/april2000/april-375-382.htm

20. Narayanan S, Parandaman V, Narayanan P, Venkatesan P, Girish C, Mahadevan S, Rajajee S. Evaluation of PCR using TRC4 and IS6110 primers in detection of tuberculous meningitis. J Clin Microbiol. 2001; 39, 2006-2008. https:// www.researchgate.net/publication/12010310

21. Radhakrishnan I, Manju Y K, Kumar R A \& Mundayoor S. Implications of low frequency of IS6110 in fingerprinting field isolates of Mycobacterium tuberculosis from Kerala, India. J ClinMicrobiol. $2001 ; 39,1683$. pubmedcentralcanada.ca/ pmcc/articles/PMC88004/pdf/jm001683.pdf

22. Bhigjee AI, Padayachee R, Paruk H, Hallwirth-Pillay $\mathrm{KD}$, Marais S, Connoly C. Diagnosis of tuberculous meningitis: Clinical and laboratory parameters. Int J Infect Dis. 2007;11(4):348-354. http://www.ijidonline.com/ article/S1201-9712(06)00190-1/fulltext

23. Rafi W, Venkataswamy MM, Ravi V, Chandramuki A. Rapid diagnosis of tuberculous meningitis: A comparative evaluation of in-house PCR assays involving three mycobacterial DNA sequences, IS6110, MPB-64 and 65 kDa antigen. J Neurol Sci. 2007;252:163-168. http:/www. jns-journal.com/article/S0022-510X(06)00529-6/fulltext

*Corresponding author:

Dr. Gargi Choudhury, Assistant Professor, Department of Microbiology, Assam Medical College, Dibrugarh, Assam, India, Pin-786002

Phone: +91 9435284089

Email: gargicb@gmail.com

Financial or other Competing Interests: None.

Date of Submission : 03.11.2017

Date of Acceptance : 12.11.2017

Date of Publication : 17.11.2017 